\section{A 21-year-old male with right chest pain, dyspnoea on exertion and bloody pleural effusion}

\section{CLINICAL PRESENTATION}

A 21-year-old man was admitted to our hospital due to right chest pain and shortness of breath on exertion for 2 months; the symptoms had been exacerbated for 4 days. He denied having cough, sputum production, chill, fever, night sweats or a history of surgery or trauma. His past history was unremarkable. On physical examination the right lung was dull on percussion and the respiratory sounds from the right inferior lung disappeared on auscultation.

A chest CT scan revealed moderate right pleural effusion; no solid lesion was identified in the lung and there were no enlarged lymph nodes in the mediastinum (figure 1); $3-\mathrm{D}$ reconstruction revealed destruction of the first to fourth ribs, as well as the middle lower part of the right scapula (figure 2).
Ultrasound-guided thoracentesis yielded $500 \mathrm{ml}$ of bloody effusion. Routine biochemical tests of the pleural effusion showed that it was exudative. Pleural fluid tests included carcinoembryonic antigen (CEA), lactate dehydrogenase (LDH) and adenosine deaminase (ADA) which were all normal; haematocrit $9.20 \%$ and haemoglobin $29 \mathrm{~g} / \mathrm{l}$. Cytology was negative for malignant cells.

\section{QUESTION}

What is your diagnosis?

See page 836 for the answer

\section{Xu Zhi, Zhang Oiao, Wang Changzheng, Wu Guoming, Wang Guansong, Yao Wei}

Respiratory Disease Department, Xinqiao Hospital, Third Military Medical University, Chongqing, PR China

Correspondence to Yao Wei, Respiratory Disease Department, Xinqiao Hospital, Chongqing 400037, PR China; wei_yao@tom.com

Competing interests None

Patient consent Obtained

Provenance and peer review Not commissioned; externally peer reviewed. Thorax 2010;65:769. doi:10.1136/thx.2010.137992
Figure 1 Chest CT scan revealed moderate right pleural effusion. No solid lesion was identified in the lung and there were no enlarged lymph nodes in the mediastinum.
Figure 2 Chest CT 3-D reconstruction revealed destruction of the bone of the middle lower part of the right scapula (A) and the right first to fourth ribs (B) (arrowed).
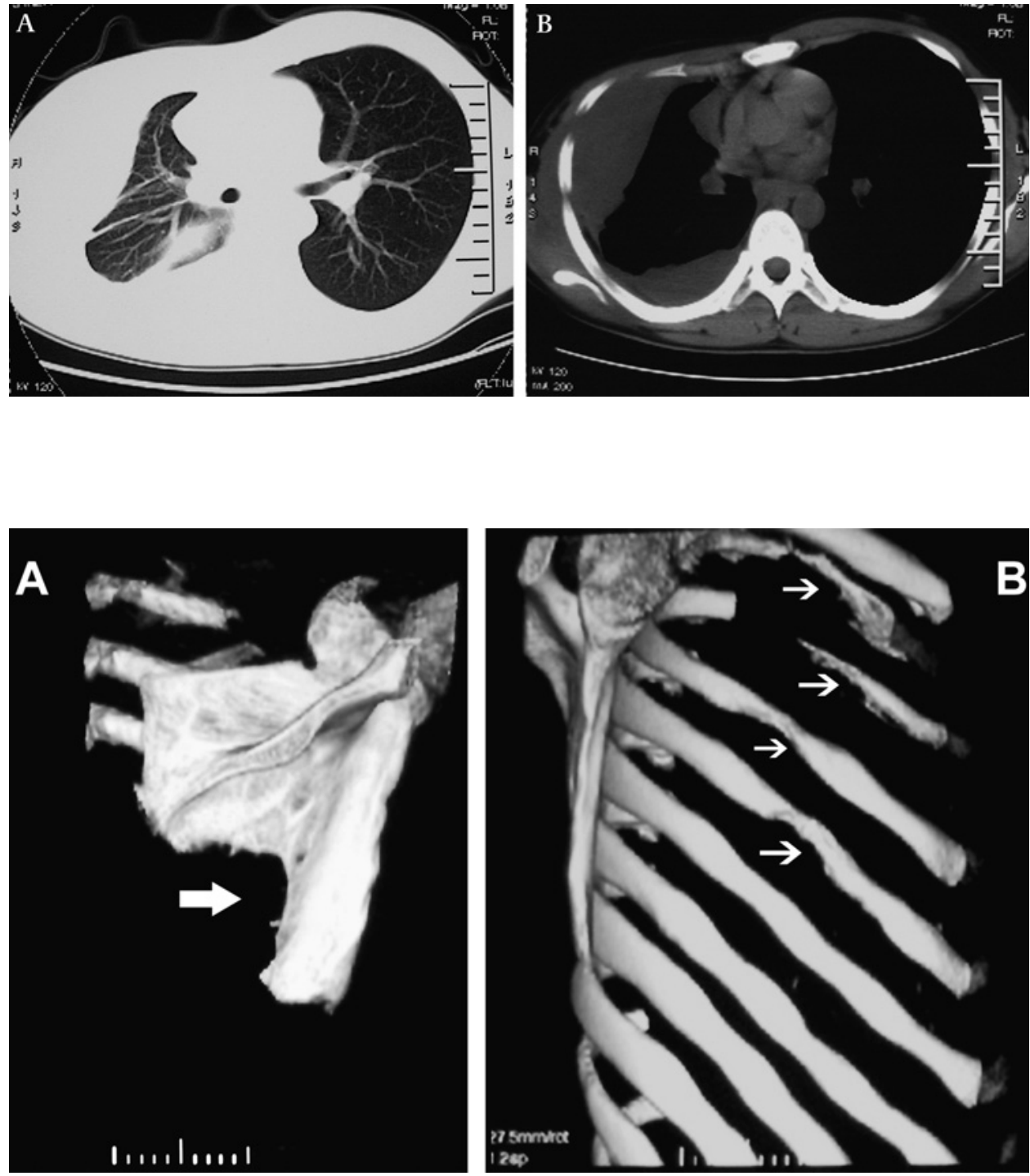


\section{ANSWER}

From the question on page 769

Thoracoscopy revealed a normal right lung with a bloodoozing lesion measuring $6 \mathrm{~cm}^{2}$ on the right lateral chest wall. The biopsy of the blood-oozing lesion revealed chronic inflammation. A pathological examination of the right scapula lesion revealed an intramuscular haemangioma in the right chest wall (figure 1).

\section{DISCUSSION}

The usual reasons for bloody pleural effusion include tumours, sarcomas, pulmonary embolism, trauma to the chest wall/diaphragm/lung/mediastinum, bleeding disorder and hereditary haemorrhagic telangiectasia, osteochondroma of the ribs, etc. Bloody pleural effusion caused by an intramuscular haemangioma has not been reported previously.

Haemangioma is a rare congenital benign lesion and is thought to be associated with an imbalance of proangiogenic factors and angiogenesis inhibitors. ${ }^{1}{ }^{2}$ Approximately 80-90\% of haemangiomas develop before the age of 30 and the disease occurs in both sexes. Chest wall haemangioma is rare and can be classified as chest intramuscular haemangioma. It can be easily misdiagnosed, and differential diagnoses include lipoma, vascular lipoma, abscess, haematoma and malignant tumours. $^{3}$

Diagnosis of haemangioma depends primarily on imaging and pathological examinations. The most widely used imaging studies include CT and MRI; angiography and MRI are the most important modalities for diagnosis of haemangioma. ${ }^{4}$ The MRI image may reveal an intramuscular irregular mass with T1weighted image intensity equal to or slightly higher than that of the skeletal muscle. ${ }^{6}$

The complications of intramuscular haemangioma depend on its size, growth location and growth rate. Complications include ulceration (skin breakdown), which can bleed or become infected; obstruction of vital functions such as vision, hearing or breathing if the intramuscular haemangioma oppresses nerve, tendon and other tissues; and, very rarely, internal bleeding or high output cardiac (heart) failure resulting from a haemangioma in an internal organ. Intramuscular haemangioma of the head and neck can induce distortion of facial features, which will lead to psychosocial complications.

The treatment of haemangioma should be individualised, depending on the location of the tumour mass, the depth of its infiltration, and the age and cosmetic requirements of the patient. Comprehensive treatment strategies are recommended, including dry ice cryotherapy, radiotherapy, steroid treatment, sclerosing agent injection, vascular ligation, vascular embolism and surgical excision. ${ }^{7} 8$

Degeneration of an intramuscular haemangioma does not tend to occur and the local recurrence rate is high; therefore, the optimal treatment is to excise the tumour mass extensively to ensure there is no residual tumour tissue.

Our patient had an intramuscular haemangioma in the right chest wall which destroyed the middle lower part of the right scapula and the right first to fourth ribs. While chest wall intramuscular haemangiomas often cause chest pain and chest mass, they do not typically cause a bloody pleural effusion as seen in this case. To our knowledge, this is the first case of a intramuscular haemangioma-associated bloody pleural effusion

Thorax 2010;65:836. doi:10.1136/thx.2010.137992a

\section{REFERENCES}

1. Atalay M, Gordillo G, Roy S, et al. Anti-angiogenic property of edible berry in a model of hemangioma. FEBS Lett 2003;544:252-7.

2. Nagasaka M, Naganuma $H$, Satoh E. Growth potential of orbital cavernous hemangioma suggested by vascular endothelial growth factor and its receptor flk-1. Neurol Med Chir (Tokyo) 2007;47:5-10.

3. Nakamura H, Miwa K, Miyoshi K, et al. Cavernous hemangioma with hematoma in the chest wall due to penetration from the anterior mediastinum. Gen Thorac Cardiovasc Surg 2007;55:184-6.

4. Barzin M, Maleki I. Incidence of vertebral hemangioma on spinal magnetic resonance imaging in Northern Iran. Pak J Biol Sci 2009;12:542-4.

5. Kuo YT, Lin MB, Sheu RS, et al. Imaging diagnosis of cavernous hemangioma of the rib-one case report and review of the literature. Gaoxiong Yi Xue Ke Xue Za Zhi 1994;10:469-73.

6. Olsen KI, Stacy GS, Montag A. Soft-tissue cavernous hemangioma. Radiographics 2004;24:849-54.

7. Ogino I, Torikai K, Kobayasi S, et al. Radiation therapy for life- or function-threatening infant hemangioma. Radiology 2001:218:834-9.

8. Hasan 0, Tan ST, Gush J, et al. Steroid therapy of a proliferating hemangioma: histochemical and molecular changes. Pediatrics 2000;105:117-20.
Figure 1 (A) Thin-walled capillary-like structure clusters with the typical pathological features of a capillary haemangioma. (B) Different sizes and an irregular vessel-like structure comprise the cavernous haemangioma.

H\&E $\times 100$.
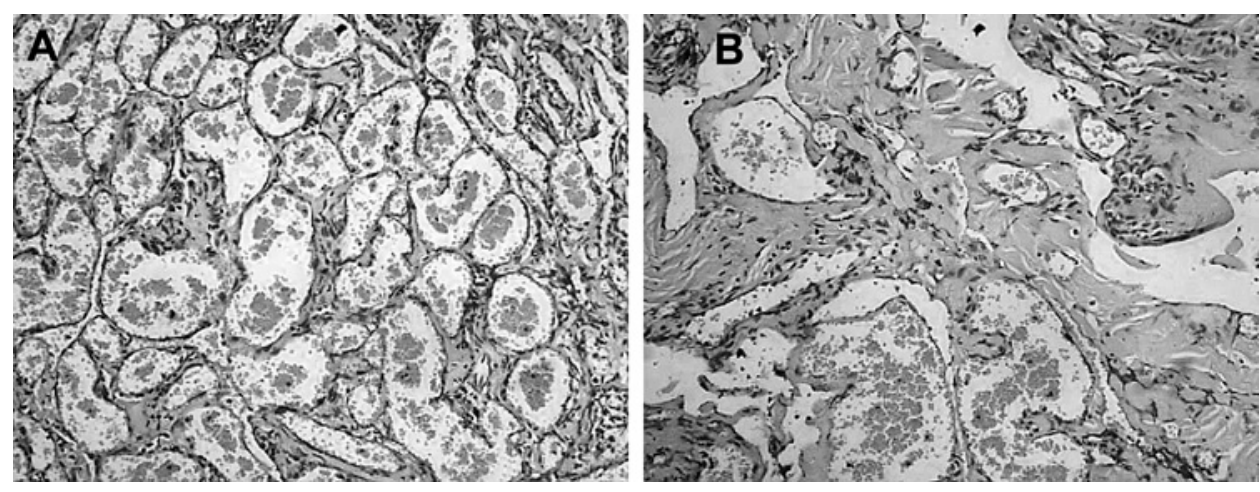